Pre-print of: Xu, Zhikun, et al. “Formulating tumor-homing peptides as regular nanoparticles enhances receptor-mediated cell penetrability”. Lliurat a Elsevier. Final version avalaible in DOI: 10.1016/j.matlet.2015.04.055

\title{
Formulating tumor-homing peptides as regular nanoparticles enhances receptor-mediated cell penetrability
}

Zhikun $\mathrm{Xu}^{\mathrm{a}, \mathrm{b}, \mathrm{c} \overline{\mathrm{T}}}$, Ugutz Unzueta ${ }^{\mathrm{c}, \mathrm{d} \overline{\mathrm{T}}}$, Mónica Roldán ${ }^{\mathrm{e}}$, Ramón Mangues ${ }^{\mathrm{c}, \mathrm{d}}$, Alejandro Sánchez-Chardi ${ }^{\mathrm{e}}$, Neus Ferrer-Miralles ${ }^{\text {a, b, c }}$, Antonio Villaverde ${ }^{a, b, c^{*}}$ and Esther Vázquez ${ }^{\text {a, b, c }}$

a Institut de Biotecnologia i de Biomedicina, Universitat Autònoma de Barcelona, Bellaterra, 08193 Barcelona, Spain;

${ }^{\mathrm{b}}$ Departament de Genètica i de Microbiologia, Universitat Autònoma de Barcelona, Bellaterra, 08193 Barcelona, Spain;

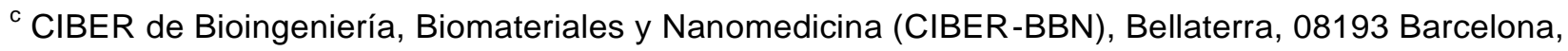
Spain,

dOncogenesis and Antitumor Drug Group and Nanotoxicology Unit, Biomedical Research Institute Sant Pau (IIB-SantPau), Hospital de la Santa Creu i Sant Pau, C/ Sant Antoni Maria Claret, 167, 08025 Barcelona, Spain,

e Servei de Microscòpia, Universitat Autònoma de Barcelona, Bellaterra, Barcelona, Spain;

${ }^{\bar{\top}}$ Equally contributed.

* Corresponding author: Antoni.Villaverde@uab.cat 


\begin{abstract}
:
Homing peptides are exploited in nanomedicine to functionalize either free drugs or nanostructured materials used as drug carriers. However, the influence of multivalent versus monovalent peptide presentation on the interaction with the receptor and on the consequent intracellular delivery of the associated cargo remains poorly explored. By using a tumor-homing peptide (T22) with regulatable selfassembling properties we have investigated here if its display in a either a monomeric form or as multimeric, self-assembled protein nanoparticles might determine the efficacy of receptor-mediated penetrability into target cells. This has been monitored by using a fluorescent cargo protein (iRFP), which when fused to the homing peptide acts as convenient reporter. The results indicate that the nanoparticulate protein versions are significantly more efficient in mediating receptor-dependent uptake than their unassembled counterparts. These finding stresses an additional benefit of nanostructured materials based on repetitive building blocks, regarding the multivalent presentation of cell ligands that facilitate cell penetration in drug delivery applications.
\end{abstract}

\title{
Keywords:
}

Biomaterials, Biomimetic, Nanoparticles, Protein materials

\section{Introduction}

Cell targeted drug delivery is expected to result in high concentration levels of drugs in desired cells and tissues, aiming to minimize side effects of chemotherapies and to thereby improve the patient's quality of life. Tumor-homing peptides specifically internalize cancer cells via specific binding to over-expressed cellsurface receptors. Therefore, they have been explored for use as targeting tools in drug delivery, either fused to therapeutic proteins [5], coupled to drugs or therapeutic nucleic acids [2,17], or as functionalizing agents of different types of nanoparticles intended as drug carriers $[1,8]$. Under the combined development of materials sciences and nanomedicine it is however unclear whether tumor-homing peptides are more effective in promoting passenger drug penetration when formulated as plain molecular preparations or as multimeric presentations on nanoparticulate entities.

Recently, we have developed a new protein engineering principle to construct protein-only nanoparticles for targeted drug and DNA delivery in cancer $[12,14,15]$. This has been possible through the construction of selfassembling building blocks containing tumor homing peptides, which organized as highly stable nanoscale entities. The size of these constructs $(>8 \mathrm{~nm}$ ) allow them to escape renal filtration [4], thus exhibiting a convenient biodistribution upon administration and exclusively accumulating in primary tumor and metastatic foci [13]. Among the set of different building blocks generated under this principle, the protein T22-IRFP-H6 exhibits unique assembling properties. In a standard physiological buffer, it organizes as toroidal nanoparticles but in presence of high salt content, the building blocks remain fully disassembled. The possibility to regulate the assembling of T22-IRFP-H6 offers an exceptional opportunity to comparatively 
explore, in the same molecular species, whether the cell targeting and internalization properties of T22, a potent ligand of CXCR4 [13], are influenced by its presentation as either free protein molecules or as regular assembled, protein-only nanoparticles.

\section{Materials and methods}

\subsection{Protein production and characterization}

T22-IRFP-H6 was produced by standard procedures [4]. After purification, the protein was collected and dialyzed against two alternative buffers: $\mathrm{NaHCO}_{3} 166 \mathrm{mM}, \mathrm{pH}=7.8$ and $\mathrm{NaHCO}_{3} 166 \mathrm{mM}, \mathrm{NaCl} 333 \mathrm{mM}$, $\mathrm{pH}=7.8$, overnight at $4 \stackrel{\circ}{\circ}$. Fluorescence emission spectrum was measured at $710 \mathrm{~nm}$, by using a JASCO FP-8000 spectrofluorometer (JASCO, US), with an excitation wavelength of $635 \mathrm{~nm}$. volume size distribution of nanoparticles and monomeric proteins were determined by dynamic light scattering (DLS, Zetasizer Nano ZS, Malvern Instruments Limited, Malvern, UK), at $633 \mathrm{~nm}$. For size exclusion chromatography (SEC) was done in a calibrated Superdex200 10/300GL (Tricorn) column (GE Healthcare).

\subsection{Cell culture, flow cytometry and competition assay}

HeLa cells (ATCC-CCL-2) were grown on 24 wells plate for $24 \mathrm{~h}$ in OptiPRO ${ }^{\text {TM }}$ SFM medium (Life technologies) supplemented with $3 \mathrm{mM} \mathrm{L-Glutamine.} \mathrm{Recombinant} \mathrm{proteins} \mathrm{were} \mathrm{added} \mathrm{to} \mathrm{each} \mathrm{well} \mathrm{at} \mathrm{a}$ final concentration of $2 \mu \mathrm{M}$ and further incubated $24 \mathrm{~h}$. After that, the medium was removed and cells washed with PBS and incubated with $1 \mathrm{mg} / \mathrm{ml}$ trypsin for $15 \mathrm{~min}$ to remove protein bound to cell surface. Cells were centrifuged at $4,000 \mathrm{~g}$ for $5 \mathrm{~min}$ to remove trypsin, and collected and resuspended in PBS. Cells were analyzed on a FACSCanto system (Becton Dickinson), using a $15 \mathrm{~W}$ air-cooled argon-ion laser at 635 $\mathrm{nm}$ excitation. IRFP fluorescence was measured with detector A (780/60 nm band pass filter). For the competition assay, AMD3100 was added $1 \mathrm{~h}$ before proteins. Protein internalization was determined $24 \mathrm{~h}$ later by flow cytometry.

\subsection{Confocal microscopy}

HeLa cells were cultured on Mat-Teck culture dishes (Mat Teck Corp., Ashland, MA, USA) with serum-free medium for $24 \mathrm{~h}$. Proteins were added with a final concentration of $2 \mu \mathrm{M}$. After $24 \mathrm{~h}$, cells were washed with PBS and nuclei and membranes stained with Hoechst 33342 and concanavalin A respectively for 10 min. After washing with PBS complete medium was added and stained cells were examined using a TCS-SP5 confocal laser scanning microscope (Leica Microsystems, Heidelberg, Germany) with a Plan Apo 63×/1.4 (oil $\mathrm{HC} \times \mathrm{PL}$ APO I blue) objective M.

\subsection{Cryo transmission electron microscopy (CryoTEM)}

Microdrops of purified proteins ( $3 \mu \mathrm{L}$ ) were deposited on Quantifoil R1.2/1.3 grids, put in liquid ethane in a Leica EM CPC and immediately placed in a Gatan cryo-transfer specimen holder. Samples were observed in a Jeol JEM 2011 transmission electron microscope, operating at $200 \mathrm{kV}$ and equipped with a CCD Gatan 895 USC 4000 camera. 


\subsection{Field emission scanning electron microscopy (FESEM)}

Microdrops $(5 \mu \mathrm{l})$ of protein sample were added to a silicon wafer and air-dried at room temperature. Native structure of samples was observed in a FESEM Zeiss Merlin operating at $2 \mathrm{kV}$ equipped with a highresolution in-lens secondary electron detector.

\section{Results and discussion}

\subsection{Features of protein materials}

Upon production in bacteria, T22-IRFP-H6 occurred as an individual molecular species of $38.6 \mathrm{kDa}$ that partially adopted a dimeric form (77.2 kDa) (Fig $1 \mathrm{~A})$, the natural organization of iRFP [6]. Further dialysis in front a physiological buffer or a buffer containing $500 \mathrm{mM}$ salt, did not alter the integrity of the protein (Fig 1B). However, in low salt buffer, T22-IRFP-H6 organized as supramolecular entities formed by around 10 monomers (Fig 1C), that were seen as regular nanoparticles of $15 \mathrm{~nm}$ by DLS (Fig 1D). Particle formation was fully confirmed by FESEM (Fig 1E) and by cryo-TEM (Fig 1F). No nanoparticles were observed when T22-IRFP-H6 was dialyzed against high salt buffer (Fig 1C-E).

\subsection{Comparative analysis of cellular internalization}

Upon approving the robustness of the analytical system we approached the comparative testing of the CXCR4-mediated cell penetrability of free T22-IRFP-H6 protein and T22-IRFP-H6 nanoparticles. We aimed to determine if the nanostructured display of the CXCR4 ligand T22 might significantly influence receptor binding and subsequent cell uptake or contrarily, if such a formulation is irrelevant regarding the performance of the tumor-homing peptide. iRFP constructs, being fully fluorescent [4], are convenient reporters in internalization assays, as free and nanostructured versions of T22-IRFP-H6 showed indistinguishable specific emission values of $13.4 \pm 0.14$ and $12.8 \pm 0.23 \mathrm{U} / \mathrm{mg}$ respectively. At the same molar ratio, both forms of T22-IRFP-H6 were internalized into CXCR4 ${ }^{+} \mathrm{HeLa}$ cells, while the control, IRFP-H6 remained extracellular (Fig 2A). However, T22-IRFP-H6 nanoparticles were internalized much more efficiently than T22-IRFP-H6 free protein, and under the same conditions, the amount of protein uptake as nanoparticles doubled that of penetrating target cells as disassembled entities.

Interestingly, the CXCR4 antagonist AMD3100, a very specific and highly potent inhibitor of CXCR4 binding $[3,7]$, blocks the entry of T22-IRFP-H6 nanoparticles more efficiently than that of soluble protein (Figure 2A). The milder blocking of disassembled protein uptake might be indicative that, not only the cell penetrability of T22 is enhanced by a nanoparticulate formulation but also the specificity of binding to the cell surface receptor. However, very low background binding of free T22-IRFP-H6 to CXCR4 SW1417 cells was also observed (Fig 2B), thereby discarding the possibility of a significant interaction of this species to an alternative receptor.

The confocal analysis of CXCR4 ${ }^{+}$HeLa cells exposed to both protein versions confirmed the enhanced cell penetrability of the nanoparticulate T22-IRFP-H6 isoform (Fig 2C). This protein version was visualized as 
discrete intracellular entities found in endosomal vesicles, free in the cytoplasm and perinuclear area (Fig 2D), and in agreement with the endosome-mediated penetration previously described for T22-empowered polypeptides [4]. Altogether, these results confirm that despite both assembled and disassembled versions of T22-IRFP-H6 internalize target cells via the tumor marker CXCR4, the penetrability of the nanostructured version is clearly higher than that of the free version. This fact is probably related to the multiple presentation of T22 on regular nanoparticles, and to the consequent multivalent receptor binding that enhances endosome formation and penetration [11], as it occurs in natural viruses [9].

\subsection{Conclusions}

In summary, we described here an enhanced performance of the tumour-homing peptide T22 as multimeric versus monomeric forms, regarding receptor-mediated cell internalization. This finding stresses the convenience to formulate targeting peptides, used for Trojan horse approaches of molecular medicine, in multivalent material arrangements. Therefore, drug delivery through carrier nanoparticles would profit not only from a higher penetrability, longer circulation time and improved stability compared to free drug versions [16], but they will also offer a convenient platform for the multiple and regular display of homing peptides. Therefore, the "artificial virus" concept in nanomedicine [10] to describe mimetic virus-like vehicles should be extended to cover not only the nanoscale nature of relevant materials, but also their potential to support a multivalent and regular display of cell binding sites in a virus-like pattern.

Acknowledgments: The authors acknowledge the financial support granted to E.V. (PI12/00327) and R.M. (PI12/01861) from FIS, to E.V. (TV32013-133930) and to R.M. and A.V. (TV32013-132031) from La Marató de TV3 (416/C/2013), to A.V. from MINECO (grant BIO2013-41019-P) and from the Centro de Investigación Biomédica en Red (CIBER) de Bioingeniería, Biomateriales y Nanomedicina (NANOPROTHER and NANOCOMETS projects). We are grateful to the Protein Production Platform (CIBER-BBN-UAB) for protein production and purification services (http://www.ciber-bbn.es/en/programas/89-plataforma-de-produccion-deproteinas-ppp), to the Servei de Cultius Cel-lulars, Producció d'Anticossos i Citometria (SCAC), to the Servei de Microscòpia, both at the UAB and to the Soft Materials Service (ICMAB-CSIC/CIBER-BBN). Z.X. received a fellowship grant from China Scholarship Council and U.U. from ISCIII. AV received an ICREA ACADEMIA award.

Figure 1. Characterization of T22-IRFP-H6-based materials. A) Western blot of purified T22-IRFP-H6 revealed with an anti-His antibody, indicating the molecular weight of markers. At the bottom, scheme of the modular composition of T22-IRFP-H6. B) Mass spectrometry of purified T22-IRFP-H6 before dialysis (BD), and after dialysis (AD) against low salt (-) and high salt buffers (+). C) SEC of T22-IRFP-H6 in low salt (green line) and in high salt (red) buffers. Figures indicate the approximate number of monomers forming the plotted structures. D) DLS plots of the same samples. E) FESEM images of T22-IRFP-H6 nanoparticles (left) at two magnification levels. No nanoparticles were observed in high salt buffer (right). F) Cryo-TEM images of T22IRFP-H6 nanoparticles at two magnification levels. 
Figure 2. Determination of CXCR4-mediated cell penetrability of T22-IRFP-H6 under alternative formulations. A) Internalized fluorescence in CXCR4 ${ }^{+}$HeLa cells. B) Penetrability into SW1417 cells overexpressing (black bars) or devoid of (grey bars) CXCR4, proteins being formulated in either low salt buffer (-) or high salt buffer (+). Protein/ AMD3100 molar ratios (either 1 or 5) are indicated (AMD1, AMD5). $\left.{ }^{*}\right), p<0.01 ;\left(^{* *}\right), p<0.001$, upon One-way ANOVA and a Tukey significance test. C) Confocal images of CXCR4+ HeLa cells incubated with protein nanoparticles (left) or monomers (right). D) 3D confocal imaging reconstruction of $\mathrm{CXCR} 4^{+}$cells internalizing nanoparticles. Cell membranes are visualized in green, nucleic acids in blue and iRFP-derived proteins in red.

\section{References}

[1] A.M. Alkilany, S.P. Boulos, S.E. Lohse, L.B. Thompson, C.J. Murphy, Homing peptideconjugated gold nanorods: the effect of amino acid sequence display on nanorod uptake and cellular proliferation, Bioconjug. Chem. 25 (2014) pp. 1162-1171.

[2] W. Arap, R. Pasqualini, E. Ruoslahti, Cancer treatment by targeted drug delivery to tumor vasculature in a mouse model, Science 279 (1998) pp. 377-380.

[3] J.A. Burger and D.J. Stewart, CXCR4 chemokine receptor antagonists: perspectives in SCLC, Expert. Opin. Investig. Drugs 18 (2009) pp. 481-490.

[4] M.V. Cespedes, U. Unzueta, W. Tatkiewicz, A. Sanchez-Chardi, O. Conchillo-Sole, P. Alamo, Z. Xu, I. Casanova, J.L. Corchero, M. Pesarrodona, J. Cedano, X. Daura, I. Ratera, J. Veciana, N. Ferrer-Miralles, E. Vazquez, A. Villaverde, R. Mangues, In Vivo Architectonic Stability of Fully de Novo Designed Protein-Only Nanoparticles, ACS Nano. 8 (2014) pp. 4166-4176.

[5] M. Cieslewicz, J. Tang, J.L. Yu, H. Cao, M. Zavaljevski, K. Motoyama, A. Lieber, E.W. Raines, S.H. Pun, Targeted delivery of proapoptotic peptides to tumor-associated macrophages improves survival, Proc. Natl. Acad. Sci. U. S. A 110 (2013) pp. 1591915924.

[6] G.S. Filonov, K.D. Piatkevich, L.M. Ting, J. Zhang, K. Kim, V.V. Verkhusha, Bright and stable near-infrared fluorescent protein for in vivo imaging, Nat. Biotechnol. 29 (2011) pp. 757-761.

[7] S. Hatse, K. Princen, G. Bridger, C.E. De, D. Schols, Chemokine receptor inhibition by AMD3100 is strictly confined to CXCR4, FEBS Lett. 527 (2002) pp. 255-262.

[8] A.R. Makela, H. Matilainen, D.J. White, E. Ruoslahti, C. Oker-Blom, Enhanced baculovirus-mediated transduction of human cancer cells by tumor-homing peptides, J. Virol. 80 (2006) pp. 6603-6611.

[9] M. Mammen, S.K. Choi, G.M. Whitesides, Polyvalent Interactions in Biological Systems: Implications for Design and Use of Multivalent Ligands and Inhibitors, Angew. Chem. Int. Ed. 37 (1998) pp. 2754-2794.

[10] E. Mastrobattista, M.A. van der Aa, W.E. Hennink, D.J. Crommelin, Artificial viruses: a nanotechnological approach to gene delivery, Nat. Rev. Drug Discov. 5 (2006) pp. 115121.

[11] S. Muro, M. Koval, V. Muzykantov, Endothelial endocytic pathways: gates for vascular drug delivery, Curr. Vasc. Pharmacol. 2 (2004) pp. 281-299.

[12] M. Pesarrodona, N. Ferrer-Miralles, U. Unzueta, P. Gener, W. Tatkiewicz, I. Abasolo, I. Ratera, J. Veciana, S.S. Jr, A. Villaverde, E. Vazquez, Intracellular targeting of CD44 cells with self-assembling, protein only nanoparticles, Int. J. Pharm. 473 (2014) pp. 286295.

[13] U. Unzueta, M.V. Cespedes, N. Ferrer-Miralles, I. Casanova, Cedano JA, Corchero JL, J. Domingo-Espin, Villaverde A, R. Mangues, Vazquez E, Intracellular CXCR4 ${ }^{+}$cell targeting with T22-empowered protein-only nanoparticles, Int. J. Nanomedicine 7 (2012) pp. 4533-4544.

[14] U. Unzueta, N. Ferrer-Miralles, J. Cedano, X. Zikung, M. Pesarrodona, P. Saccardo, E. Garcia-Fruitos, J. Domingo-Espin, P. Kumar, K.C. Gupta, R. Mangues, A. Villaverde, E. Vazquez, Non-amyloidogenic peptide tags for the regulatable self-assembling of protein-only nanoparticles, Biomaterials 33 (2012) pp. 8714-8722.

[15] U. Unzueta, P. Saccardo, J. Domingo-Espin, J. Cedano, O. Conchillo-Sole, E. GarciaFruitos, M.V. Cespedes, J.L. Corchero, X. Daura, R. Mangues, N. Ferrer-Miralles, A. 
Villaverde, E. Vazquez, Sheltering DNA in self-organizing, protein-only nano-shells as artificial viruses for gene delivery, Nanomedicine. 10 (2014) pp. 535-541.

[16] Villaverde A, Nanoparticles in Translational Science and Medicine, Academic Press (Elsevier), London, 2011.

[17] W. Yang, D. Luo, S. Wang, R. Wang, R. Chen, Y. Liu, T. Zhu, X. Ma, R. Liu, G. Xu, L. Meng, Y. Lu, J. Zhou, D. Ma, TMTP1, a novel tumor-homing peptide specifically targeting metastasis, Clin. Cancer Res. 14 (2008) pp. 5494-5502. 